\title{
Methane/Oxygen Combustion Kinetic Scheme Optimization for Liquid Rocket Engine CFD Applications
}

\author{
Guido Saccone ${ }^{1}$, Pasquale Natale ${ }^{1}$, Francesco Battista ${ }^{1}$, Paola Breda ${ }^{2}$, Michael Pfitzner ${ }^{2}$ \\ ${ }^{1}$ Italian Aerospace Research Centre - CIRA \\ Via Maiorise, Capua, Italy \\ g.saccone@cira.it; p.natale@cira.it; f.battista@cira.it; \\ ${ }^{2}$ Universität der Bundeswehr München \\ 85577 Neubiberg, Germany \\ paola.breda@unibw.de
}

\section{Extended Abstract}

The research and development process involved in the production of Liquid Rocket Engines (LRE) for space access poses several challenges to CFD simulations of turbulent reactive flows. As the computational cost should be minimized, reduction of reaction mechanisms appears necessary, even more if the combustion involves hydrocarbons. Therefore, derivation of a suitable skeletal, kinetic mechanism for the selected fuel and oxidizer, conveniently tailored on the operative temperature, pressure and mixture ratio of the LRE under development, is appealing to obtain accurate results in a reasonable time.

In this work, two skeletal kinetic schemes characterizing methane/oxygen ignition, combustion and afterburning, were derived from the detailed GRIMECH 3.0 [1] using the open-source OD chemical-kinetic software CANTERA [2], with both Python and MathWorks Matlab interfaces. The procedure involves the identification of the reaction paths, a sensitivity analysis and a final tuning [3], [4]. These schemes were preliminary analysed in terms of ignition delay times and peak flame temperatures for $0 \mathrm{D}$ simulations of homogeneous, isobaric, adiabatic, closed reactors and for temperature profiles of 1D counterflow diffusion flames. Promising results were achieved in the development.

The first scheme, referred as LPRB, was generated for atmospheric pressure, temperature of $2000 \mathrm{~K}$ and stoichiometric composition. It was tested with ANSYS - Fluent in a 2D axisymmetric simulation of the exhaust gases plume exiting a LRE. The calculation reveals not only a run-time speed-up of 3.8 compared to the full mechanism, but also an agreement on the longitudinal axis of pressure, temperature and the main chemical species profiles. Their root mean square (RMS) values compared to the full mechanism are larger than 0.9. The second scheme, labelled as MPRB, was conceived for pressures close to 20 bar, low-intermediate temperatures of about $1000-1200 \mathrm{~K}$ and fuel-rich composition and it was validated against counterflow laminar flames.

Both mechanisms were used to generate non-adiabatic flamelet manifolds as reported in [5], where the fluid thermochemical properties are pre-tabulated in order to speed up the CFD computation. The CPU time obtained from a single flamelet calculation with the new schemes is compared to run time of mechanism Lu30 (skeletal from GRI3.0). A 2D methane-oxygen laminar flame parallel to an isothermal wall in a 20 bar environment is calculated in OpenFOAM, and serves as benchmark for assessing the performance and accuracy of the new schemes. The results show a similar behaviour for LPRB and MPRB, which both predict higher recombination of the main products $\mathrm{CO}, \mathrm{H}_{2}$ at wall compared to the Lu30. This is also reflected in an increased wall heat flux due to the shifting of the chemistry towards the exothermic reactions. The $\mathrm{H}_{2} \mathrm{O}$ concentration at wall is well captured by the LPRB, while it is overestimated by the MPRB. In the core flow, where the flame is not affected by the heat losses due to the isothermal wall, both schemes agree very well with the Lu30.

\section{References}

[1] G. P. Smith, D. M. Golden, M. Frenklach, N. W. Moriarty, B. Eiteneer, M. Goldenberg, C. T. Bowman, R. K. Hanson, S. Song, W. C. Gardiner, Jr., V. V. Lissianski, and Z. Qin [Online].

Available: http://www.me.berkeley.edu/gri_mech/ 
[2] D. Goodwin, H. K. Moffat, R. L. Speth. (2017). Cantera: An Object-oriented Software Toolkit for Chemical Kinetics, Thermodynamics, and Transport Processes (2.6 version) [Online]. Available: http://www.cantera.org

[3] G. Saccone, P. Natale, F. Battista, "Reduced Kinetic Scheme of Methane/Oxygen Combustion for Liquid Rocket Engines Applications", Proceedings of the $3^{\text {rd }}$ World Congress on Momentum, Heat and Mass Transfer (MHMT'18), Budapest, Hungary, 2018.

[4] V. P. Zhukov, A. F. Kong, "Skeletal Kinetic Mechanism of Methane Oxidation for High Pressures and Temperatures," in Proceedings of $7^{\text {th }}$ European Conference for Aeronautics and Space Sciences (EUCASS), 2015.

[5] P. Breda, J. Zips, M. Pfitzner, "A Non-Adiabatic Flamelet Approach for Non-Premixed $\mathrm{O}_{2}-\mathrm{CH}_{4} \mathrm{Combustion",}^{-}$ Proceedings of the $3^{\text {rd }}$ World Congress on Momentum, Heat and Mass Transfer (MHMT'18), Budapest, Hungary, 2018. 